\title{
Familial breast cancer
}

\author{
R.F. Phipps and P.M. Perry \\ Queen Alexandra Hospital, Cosham, Portsmouth, Hants PO6 3LY, UK.
}

\section{Review Article}

Summary: Familial breast cancer is important because of all the known risk factors associated with developing the disease. The one with the most predictability is a positive family history. It is also important because a family history causes anxiety in the families concerned, and young women will often ask their chance of developing the disease. This form of breast cancer accounts for $10 \%$ of causes and has factors that distinguish it from the sporadic variety. Relatives of familial breast cancer patients are not only at an increased risk of developing the disease (if female) but also of developing other tumours especially colonic.

\section{Introduction}

Breast cancer is the commonest malignancy to affect women in the western nations and accounts for 14,000 deaths in the United Kingdom and 40,000 in America each year. There are two main types of breast cancer, the sporadic variety which accounts for $90 \%$ of patients and the familial type which accounts for the remaining $10 \%$. Of all the risk factors known to be associated with developing breast cancer, the one with the most predictability is a positive family history of the disease. Because of the greater patient awareness of the importance of a positive family history, familial breast cancer will assume greater clinical importance than in the past. This review will elucidate the features of familial breast cancer that separate it from the sporadic variety of the disease and denotes the risk for developing breast cancer.

\section{Definition}

Familial breast cancer is defined as the occurrence of two of more first degree relatives in the nuclear pedigree including the proband. ${ }^{1}$ The proband is the individual or patient from whom the family history is traced.

Correspondence: R.F. Phipps, M.B., B.Ch., F.R.C.S., 27 Ancaster Crescent, Mew Malden, Surrey KT3 6BD, UK. Accepted: 19 May 1988

\section{Historical review}

The first significant report concerning the genetics of breast cancer was by Broca in 1866, who traced the cause of death of 38 members of his wife's family through five generations. He also documented all other malignant neoplasms which included an excess of cancer of the gastrointestinal tract. Broca's observations were later substantiated by Jacobsen when he found an increased frequency of breast cancer in female relatives of breast cancer probands. He also found an increased incidence of cancer at all sites among relatives. ${ }^{2}$ Since then familial clustering has been verified repeatedly in other studies.

\section{Population screening}

Two recent studies in Sweden and America have shown a $30 \%$ increased survival in mammographically screened women. ${ }^{3,4}$ We also know that the 5 year survival of node negative patients is $80 \%$ which drops to $45 \%$ in node positive patients. These facts support any effort to detect early breast cancer.

Population screening for breast cancer is to be implemented in this country for women over 50 and provision has been made for women under 50 who have a positive family history of breast cancer. ${ }^{5}$ Familial breast cancer as defined identifies a group of women, i.e. the relatives of patients, who are at an increased risk, and screening of this group should provide a greater reward.

(C) The Fellowship of Postgraduate Medicine, 1988 


\section{Features of familial breast cancer}

Familial breast cancer differs from its sporadic counterpart by the following features: (1) significant early age of onset; (2) excess bilaterality; (3) specific tumour associations; (4) vertical transmission; (5) improved survival over sporadic form of the disease; (6) pregnancy at an early age confers no protection.

\section{Early age of onset}

This is a feature of all varieties of hereditary cancer including breast. ${ }^{6}$ It has been shown that only $1.8 \%$ of breast cancers are found in women under 30 years of age. ${ }^{7}$ Lynch studied 52 breast cancer families and observed $6.9 \%$ of patients under 30 and in $4.1 \%$ cancer was present under the age of 25.

Another observation was the earlier age of diagnosis among daughters of breast cancer affected mothers who were members of families showing hereditary breast cancer.

\section{Bilateral breast cancer}

Bilateral breast cancer and an excess of multiple primary cancer (various sites) is an integral feature of hereditary breast cancer. Anderson has shown a five-fold increase in the number of bilateral tumours in patients with the familial form of the disease. Bilateral breast cancer is generally present in $3 \%$ of patients and in his study of 500 patients with a positive family history the bilateral rate was $9.6 \% .^{1}$ The difference between familial and nonfamilial patients became more apparent when familial patients were classified according to their ages at diagnosis: early - this is when the disease was diagnosed between the ages of 20 and 44 years; intermediate - this is when the disease is diagnosed between 45 and 54 years, and late, this is when the diagnosis occurred after 55 years. In the familial group of patients with an early diagnosis these exhibited a frequency of bilaterality of $15.5 \%$, which was significantly higher than the $4.8 \%$ in late cases.

A further study of 198 patients from 75 cancerprone pedigrees showed a cumulative risk to the contralateral breast in patients whose first breast cancer was diagnosed pre-menopausally, to be $46.4 \%$ over a 20 year period. ${ }^{8}$

\section{Specific tumour association}

Jacobsen was one of the first investigators to question the inheritance of breast cancer syndrome as being solely site-specific. He found an increased frequency of cancer of all sites in the first degree 3 relatives of the probands. ${ }^{2}$ The most frequent $\stackrel{\varnothing}{2}$ reported associations are breast and colon and $C$ breast and ovary. A statistical association of cancer $\overrightarrow{\vec{F}}$ of the breast and the large bowel has been provided by Schoenberg, who observed a higher than expected frequency of 2 nd primary malignant neoplasms of the large bowel when the first cancer involved the breast. ${ }^{9}$ One study identified 34 breast cancer families and revealed that gastrointestinal cancer was present in 22 of these. ${ }^{10}$ The increased $\vec{\circ}$ incidence of colon cancer found in first degree relatives may be an indication of the putative hereditary breast cancer syndrome, i.e. familial breast cancer patients without associated gastrointestinal cancer may not be categorized as true hereditary breast cancer syndrome.

\section{Vertical transmission}

By the use of statistical methods such as segrega- $\frac{}{3}$ tion analysis the mode of transmission of breast cancer genotype is in accordance with an autosomal dominant pattern. ${ }^{11}$ The gene or genes may be transmitted through either paternal or maternal lineages, though males are rarely affected. The $\mathbb{Q}$ expression of the cancer phenotype is dependent io part upon the penetrance of the gene and possibfe exposure to specific carcinogenic promotors.

\section{Improved survival}

In one study the five year disease-free survival rate was found to be $66 \%$ in the hereditary form of the disease, which was significantly better than the $42 \%$ survival in the American College of Surgeons (A.C.S.) Register Study. ${ }^{12}$ It remains obscure why this should be so, but it was not felt that earlier diagnosis was a factor or that the patients were on average younger than the comparable A.C.S. Tumour Registry patients.

\section{Lack of a protective effect of pregnancy at an early age}

A pregnancy at an early age is believed to have a protective effect in the sporadic form of breast cancer, but this has been shown not to be the case in familial breast cancer. ${ }^{13}$

\section{What is the importance of familial breast cancer?}

This is a question of critical importance and is best answered by stating the risk of developing the disease. Anderson showed significant variation in breast cancer risk to first degree relatives of cancer 
probands. ${ }^{14}$ Specifically he compared breast cancer patients who had a family history of breast cancer with controls consisting of cancer patients with family histories of malignant neoplasms arising in sites other than the breast. He concluded that first degree relatives of breast cancer patients manifest a two- to three-fold increased risk of developing breast cancer. When the disease in the patient was bilateral the risk for the relatives was five-fold and if the disease was both premenopausal and bilateral the risk was increased nine-fold. No increased risk was observed for relatives of patients with a postmenopausal diagnosis.

In a further study Schwartz estimated the cumulative risk of breast cancer before the age of 50 to be $1 \%$ for relatives of controls, $3 \%$ for sisters of older cases, but $17 \%$ for women with sisters diagnosed before the age of 40 years or with an affected sister and mother. Sisters of older patients had a $15 \%$ risk of breast cancer by the age of 70 years compared to sisters of controls, who had a risk of $5 \%$. Women with an affected mother and sister had a $50 \%$ chance of developing the disease by the age of 65.15

In other studies the estimated life time risk of an offspring of patients with familial breast cancer developing breast cancer or another associated

\section{References}

1. Anderson, D.E. Some characteristics of familial breast cancer. J Natl Cancer Inst 1971, 28: 1500-1504.

2. Jacobsen, $\mathrm{O}$. Heredity in breast cancer, a genetic and clinical study of two hundred probands. H.K. Lewis, London, 1946.

3. Tabar, L., Fagerberg, C.J.G., Gao, A. et al. Reduction in mortality from breast cancer after mass screening with mammography. Lancet 1985, i: 829-832.

4. Shapiro, S., Venet, W., Shax, P., Venet, L. \& Roese, R. Ten to fourteen year effect of breast screening on breast cancer mortality. J Natl Cancer Inst 1982, 69: 349-355.

5. Forest, P. Report of the UK. Working Group on Breast Cancer Screening. H.M.S.O., London, 1986.

6. Lynch, H.T., Guirgis, H.A., Brodkey, F. et al. Early age of onset in familial breast cancer: genetic and cancer control implications. Arch Surgery 1976, III: 126-131.

7. Haagensen, C.D. Disease of the Breast. W.B. Saunders, Philadelphia, 1956.

8. Harris, R.E., Lynch, H.T. \& Guirgis, H.A. Familial breast cancer-risk to the contralateral breast. $J$ Natl Cancer Inst 1978, 60: 955-960.

9. Schoenberg, B.S., Greenberg, R.A. \& Eisenberg, H. Occurrence of certain multiple primary cancer in females. J Natl Cancer Inst 1969, 43: 15-32. malignancy was approximately $50 \%$ in females and $42 \%$ in males. ${ }^{16}$ From these figures it would appear that identifying the familial form of the disease is of importance for the specific family and also has important implications for populaton screening for other cancers. Finally, Lynch studied 328 patients and showed that a more intensive family history documentation in patients about doubles the familial category. ${ }^{17}$ This would strongly suggest an underestimation of the magnitude of the host factor component in breast cancer aetiology.

\section{Conclusion}

The risk of breast cancer to women with a family history of this disease remains a concern for breast cancer patients, their relatives and clinicians. Women with a positive family often ask what their risk is of developing the disease. The figures are available but more importantly the risk of developing cancer extends to other forms of cancer, especially colonic. At present risk can only be inferred from a personal history as no biological or genetic marker has been identified that will in turn identify women at increased risk.

1.0. Lynch, H.T., Krush, A.J., Guirgis, H.A. et al. Genetic factors in families with combined gastrointestinal and breast cancer. Am J Gastroenterol 1973, 59: 31-40.

11. Go, R.C.P., King, M.-C., Bailey-Wilson, J. et al. Genetic epidemiology of breast cancer associated cancer on high risk families. 1 . Segregation analysis. $J$ Natl Cancer Inst 1983, 3: 455-461.

12. Albano, W.A., Recabaren, J. Lynch, H.T. et al. Natural history of hereditary cancer of the breast and colon. Cancer 1982, 50: 360-363.

13. Lynch, H.T., Albano, W.A. \& Layton, M.A. Breast cancer genetics and age at first pregnancy. $J$ Med Genet 1984, 21: 96-98.

14. Anderson, D.E. A genetic study of human breast cancer. J Natl Cancer Inst 1972, 48: 1029-1034.

15. Schwartz, A.G., King, M.-C., Bell, S.H. et al. Risk of breast cancer to relatives of young breast cancer patients. J Natl Cancer Inst 1985, 75: 665-668.

16. Lynch, H.T., Albano, W.A., Danes, B.S. et al. Genetic predisposition to breast cancer. Cancer 1984, 53: $612-627$

17. Lynch, H.T. \& Lynch, J.F. Short communication. Breast cancer genetics in an oncology clinic. 328 Conservative patients. Cancer Genet Cytogenet 1986, 22: $369-371$ 